\title{
miR-886-3p Levels Are Elevated in Friedreich Ataxia
}

\author{
Lata H. Mahishi, ${ }^{1,2}$ Ronald P. Hart, ${ }^{3}$ David R. Lynch, ${ }^{4}$ and Rajiv R. Ratan ${ }^{1,2}$ \\ ${ }^{1}$ Department of Neurology and Neuroscience, Weill Medical College of Cornell University, New York, New York 10065, ${ }^{2}$ Burke Cornell Medical Research \\ Institute, White Plains, New York 10605, ${ }^{3}$ Rutgers Stem Cell Research Center and William Myron Keck Center for Collaborative Neuroscience, Rutgers \\ University, Piscataway, New Jersey 08854, and ${ }^{4}$ Department of Neurology, Children's Hospital of Philadelphia, Philadelphia, Pennsylvania 19104
}

Friedreich ataxia (FRDA) is the most common inherited ataxia caused primarily by an intronic GAA.TTC triplet repeat expansion in the frataxin $(F X N)$ gene. FXN RNA and protein levels are reduced in patients leading to progressive gait and limb ataxia, sensory loss, reduced tendon reflexes, dysarthria, absent lower limb reflexes, and loss of position and vibration sense. Neurological manifestations ensue from primary loss of dorsal root ganglia neurons and their associated axons ascending centrally in the spinal cord and peripherally in large myelinated nerves. Small noncoding RNAs such as microRNAs have been shown to be dysregulated in neurodegenerative diseases such as Alzheimer's and Huntington's disease. Here we report that hsa-miR-886-3p (miR-886-3p) was increased in patient cells as well as peripheral patient blood samples. Selective reduction in miR-886-3p by an anti-miR led to elevation of FXN message and protein levels without associated changes in histone marks at the FXN locus. Nevertheless, derepression of frataxin by a histone deacetylase inhibitor leads to a decrease in miR-886-3p. These results outline involvement of a small RNA, miR-886-3p in FRDA and a novel therapeutic approach to this disease using an anti-miR-886-3p.

\section{Introduction}

Friedreich ataxia (FRDA) is a devastating orphan disease. It is the most common autosomal hereditary ataxia. Along with the central and peripheral nervous system, heart and pancreas are also affected (Harding, 1981) in patients. Cardiac dysfunction is the predominant reason for mortality in affected individuals (Tsou et al., 2011). Approximately $10 \%$ of patients develop diabetes. Among affected individuals, the Caucasian and South Asian populations are overrepresented. In most cases the disease is due to GAA.TTC repeat expansion in the first intron of the frataxin $(F X N)$ gene located on chromosome 9q13. In the general population the repeat length is $\leq 30$ while in patients it ranges from 66 to 1700 (Campuzano et al., 1996; Cossée et al., 1997; Montermini et al., 1997). Less than 5\% of patients with FRDA are compound heterozygotes with the GAA repeat in one allele and a frataxin point mutation (missense, nonsense, or intronic) in the other allele (Cossée et al., 1999). This GAA.TTC repeat expansion leads to decreased levels of frataxin mRNA and protein. Since the small amount of protein formed in patients is normal, increasing expression via transcriptional or translational targets should help ameliorate disease symptoms.

microRNAs (miRNAs) are small noncoding RNAs of 21-23 nt in length. They represent a relatively new family of regulatory

\footnotetext{
Received Dec. 30, 2011; revised April 20, 2012; accepted May 22, 2012.

Author contributions: L.H.M. designed research; L.H.M., D.R.L, and R.P.H. performed research; R.R.R. contributed unpublished reagents/analytictools; L.H.M., D.R.L., and R.P.H. analyzed data; L.H.M. and R.R.R. wrote the paper.

These studies were supported by Goldsmith Foundation and Friedreich Ataxia Research Alliance (FARA) grants to L.H.M. and Miriam and Sheldon G. Adelson Foundation grant to R.R.R. The patient data collection by D.R.L. was supported by the Muscular Dystrophy Association and FARA. We thank Dr. Giovanni Manfredi for useful discussions and Jennifer Farmer at FARA for her help with the sample collections.

Correspondence should be addressed to Lata H. Mahishi, Department of Neurology and Neuroscience, Weill Medical College of Cornell University, New York, New York 10065. E-mail: lam2022@med.cornell.edu.

DOI:10.1523/JNEUROSCI.0059-12.2012

Copyright $\odot 2012$ the authors $\quad 0270-6474 / 12 / 329369-05 \$ 15.00 / 0$
}

RNAs with broad potential relevance in the regulation of developmental and disease pathways. In most cases miRNAs have been shown to be negative regulators of gene expression. However, there are instances where they have been reported to be positive regulators (Place et al., 2008). Various miRNAs have been implicated in neurodegeneration and neuroprotection. Specifically, miRNA dysregulation has been reported in Huntington's disease and Alzheimer's disease (Cogswell et al., 2008; Johnson et al., 2008; Sonntag, 2010).

Despite intense focus on frataxin transcription and translation as potential therapeutic strategies, information about frataxin regulation by miRNAs or miRNAs misregulated in Friedreich ataxia have not been reported. The only in silico datum available for miRNA involvement in the FRDA paradigm is for a single-nucleotide polymorphism (SNP) at a potential miR-155 binding site in angiotensin II type 1 receptor (AGTR1). This SNP may modify the FRDA cardiac phenotype independently of the number of GAA repeats on the smaller FXN allele (Kelly et al., 2011).

\section{Materials and Methods}

Cell lines and blood samples. Cells from individuals without FRDA (lymphoblast: GM15851, Fibroblast: GM08402) and with FRDA (lymphoblast: GM15850; Fibroblast: GM04078 and GM03816) were obtained from the Coriell Cell Repository (Camden, NJ). Lymphoblasts were grown in RPMI 1640 medium supplemented with $10 \%$ fetal calf serum under standard conditions (Invitrogen Life Technologies Inc.). Fibroblasts were grown in MEM supplemented with 10\% certified fetal bovine serum under standard conditions (Invitrogen Life Technologies Inc.). The blood samples were taken after informed consent. The blood samples were collected in Paxgene blood RNA tubes (PreAnalytiX).

miRNA expression profiling using TaqMan MiRNA assays. Total RNA was isolated from lymphoblasts using TRIzol (Invitrogen Life Technologies Inc.) using the manufacturer's instructions. RNA integrity was assessed by Bioanalyzer (Agilent) and concentrations determined by 
Nanodrop spectrophotometry. The RNA was reverse transcribed using Megaplex Primer Pools and TaqMan MicroRNA Reverse Transcription Kit (Applied Biosystems by Life Technologies). Microarray was performed using TaqMan Human MicroRNA Array Set v2.0 (Applied Biosystems by Life Technologies). The U6 SnRNA was used as an endogenous control. The level in control cell line was set at 1 .

RNA isolation and quantitative real-time PCR. Blood miRNA was isolated using PAXgene blood miRNA kit (PreAnalytiX). Total RNA was isolated from lymphoblasts and fibroblasts using TRIzol (Invitrogen Life Technologies Inc) using the manufacturer's instructions. Real-time PCR analysis was performed using TaqMan RNA-to-CT 1-Step Kit (Applied Biosystems by Life Technologies). $\beta$-Actin was use as an endogenous control. For miRNA expression analysis, total RNA was used as a source of miRNA in case of lymphoblasts and fibroblasts. For blood samples, miRNA was isolated using PAXgene blood miRNA kit per the manufacturer's instructions. The reverse transcription was done using TaqMan MicroRNA Reverse Transcription Kit (Applied Biosystems by Life Technologies) or miScript Reverse Transcription Kit (Qiagen). Quantitative real-time PCR was performed using TaqMan microRNA assay (Applied Biosystems by Life Technologies) or miScript SYBR Green PCR Kit (Qiagen).

Cell transfections. Fibroblasts were transfected with Lipofectamine 2000 (Invitrogen by Life Technologies) per the manufacturer's instructions. The cells were harvested for RNA isolation $48 \mathrm{~h}$ post transfection. Anti-miR-886 was purchased from Applied Biosystems by Life Technologies.

Western blot. Total cell extracts were prepared using M-PER mammalian protein extraction reagent (Thermo Fisher Scientific) per the manufacturer's instructions. The Samples were boiled in Laemmli buffer and electrophoresed under reducing conditions on NuPAGE Novex $4-12 \%$ Bis-Tris Gel polyacrylamide gels (Invitrogen). Proteins were transferred to a nitrocellulose membrane (Bio-Rad) by electroblotting. Nonspecific binding was inhibited by incubation in Odyssey blocking buffer (LI-COR Biosciences). Antibodies against Frataxin (MSF01; Mitosciences), GAPDH (G9545; Sigma-Aldrich) and cytochrome $c$ were used in Odyssey blocking buffer and the membranes were incubated overnight at $4^{\circ} \mathrm{C}$. Fluorophore-conjugated Odyssey IRDye-680 or IRDye-800 secondary antibody (LI-COR Biosciences) was used in Odyssey blocking buffer and membranes were incubated for $1 \mathrm{~h}$ at room temperature. Finally, proteins were detected using an Odyssey infrared imaging system (LI-COR Biosciences).

Chromatin Immunoprecipitation. The EzMagna ChIP assay kit (Millipore) was used per the manufacturer's instructions. Anti-H3K9 dimethyl $\mathrm{Ab}$ and anti-H3K36 trimethyl Ab were purchased from Millipore and Abcam, respectively. The primers used include FXNchipH3K9-F 5'-GCAAAGGCCAGGAAGGCGGA-3' (Greene et al., 2007); FXNchip-H3K9-R 5'-AT GGCTTGGACGTGGCCTGC-3' (Greene et al., 2007); FXNchip-H3K36-F 5'-ACTGTAAGCAG GCATCAGGATATTAA-3' (Punga and Bühler, 2010) and FXNchip-H3K36-R 5'-ACTGTCTAGGCTGGATTTGGAAAA G-3' (Punga and Bühler, 2010).

\section{Results}

To evaluate miRNAs in FRDA, we compared miRNA profiles between a control and a patient lymphoblast. miRNA expression array studies revealed that 27 miRNAs were dysregulated ( $\geq 2$ fold increase or decrease) in the FRDA lymphoblast (Table 1). Of these 27 miRNAs, many of which may be false positives, we further studied one miRNA, hsa-miR-886-3p (miR-886-3p). We confirmed increased expression levels of miR-886-3p by realtime PCR in the patient lymphoblast that was used for unbiased microarray analysis (GM15850) and in an additional patient fi-
Table 1. miRNA expression changes in FRDA patient lymphoblast cells as determined by microarray

\begin{tabular}{ll}
\hline MicroRNA & Expression levels (affected/unaffected) \\
\hline hsa-miR-886-3p & 4.86 \\
hsa-miR-886-5p & 5.07 \\
hsa-miR-923 & 4.32 \\
hsa-miR-518f & 4.18 \\
hsa-miR-363 & 3.46 \\
hsa-miR-21* & 3.32 \\
hsa-miR-124 & 3.14 \\
hsa-miR-130a & 2.89 \\
hsa-miR-10a & 2.86 \\
hsa-miR-505* & 2.75 \\
hsa-miR-643 & 2.71 \\
hsa-miR-155 & 2.57 \\
hsa-miR-628-3p & 2.44 \\
hsa-miR-194 & 2.37 \\
hsa-miR-20b & 2.2 \\
hsa-miR-192 & 2.14 \\
hsa-miR-181a & 2.13 \\
hsa-miR-9 & 0.47 \\
hsa-miR-212 & 0.44 \\
hsa-miR-126 & 0.37 \\
hsa-miR-335* & 0.32 \\
hsa-miR-125b & 0.28 \\
hsa-miR-9* & 0.24 \\
hsa-miR-132 & 0.18 \\
hsa-miR-944 & 0.12 \\
hsa-miR-614 & 0.08 \\
hsa-miR-500* & 0.02 \\
\hline
\end{tabular}

U6SnRNA was used an endogenous control and the miRNA levels obtained in unaffected control cells were set at 1 . An asterisk denotes the less predominant form of miRNA (Griffiths-Jones, 2004).

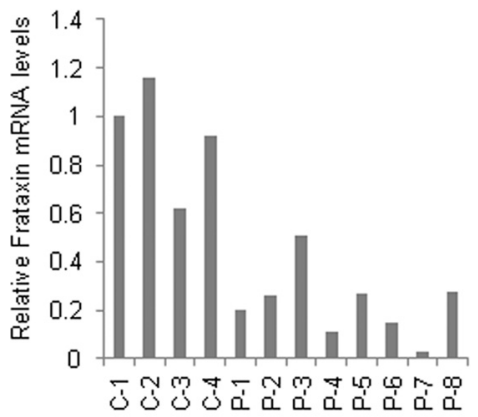

Peripheral blood samples b

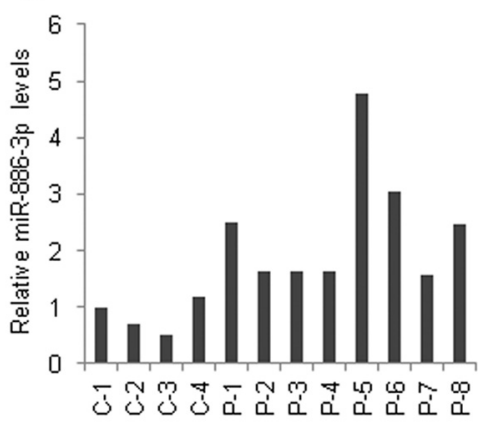

Figure 1. $\quad \boldsymbol{a}$, Frataxin mRNA levels in FRDA patient peripheral blood samples. $\beta$-Actin was used as an endogenous control and the frataxin levels obtained in control-1 sample was set at 1. $\boldsymbol{b}$, miR-886-3p expression levels in FRDA patient peripheral blood samples. U6SnRNA was used as an endogenous control and the miRNA levels obtained in unaffected control-1 were set at 1. 
a

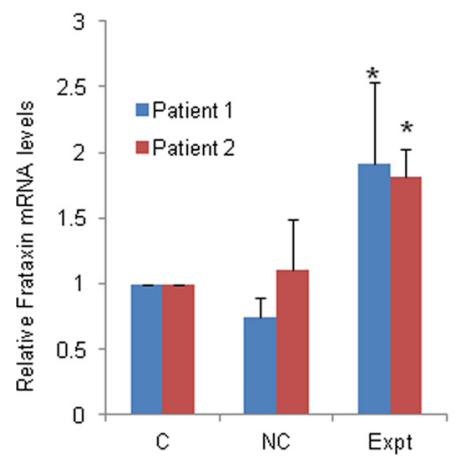

C

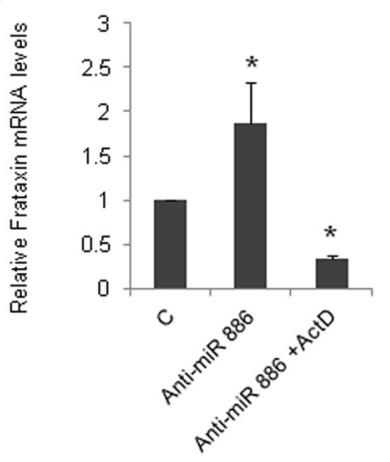

b

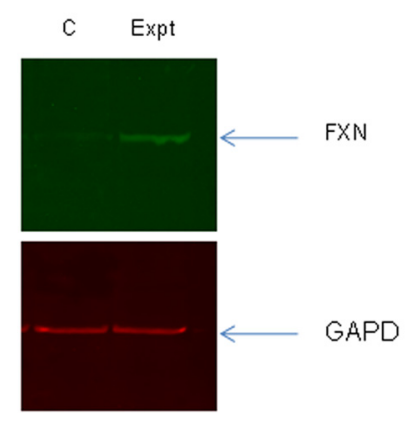

d

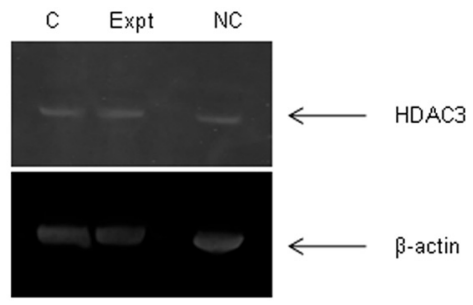

Figure 2. $\quad \boldsymbol{a}$, Frataxin mRNA levels in untransfected patient cells GM04078 and GM03816 (C), following transfection with negative control anti-miR (NC) and anti-miR-886-3p (Expt). $\beta$-Actin was used as an endogenous control for RNA levels, and frataxin mRNA levels in cells without transfection were set at $1\left({ }^{*} p<0.05\right)$. $\boldsymbol{b}$, Frataxin protein levels in untransfected cells $(C)$ and following transfection with anti-miR-886-3p (Expt) in patient cells (GM04078). GAPD was used as a loading control for protein levels. $c$, Frataxin mRNA levels following transfection with anti-miR-886-3p alone and in the presence of transcription inhibitor actinomycin D. $\beta$-Actin was used as an endogenous control, and frataxin levels in patient cells without transfection were set at 1 $\left({ }^{*} p<0.05\right)$. d, HDAC3 protein levels in untransfected cells (C), and following transfection with negative control anti-miR (NC) and anti-miR-886-3p (Expt) in patient cells. $\beta$-Actin was used as an endogenous control.

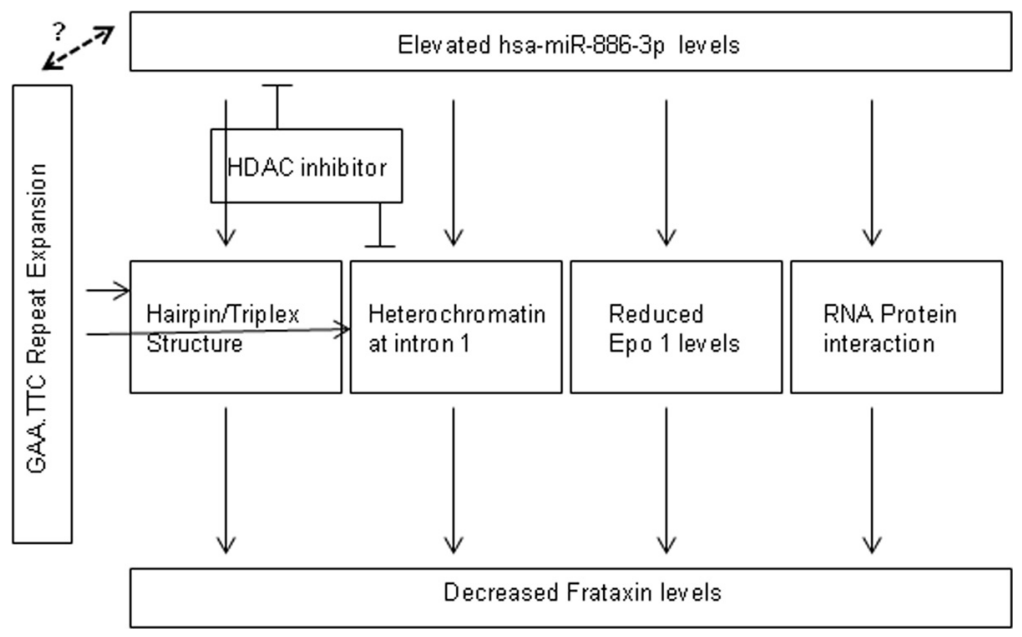

Figure 3. Potential mechanisms for involvement of miR-886-3p in FRDA paradigm.

fection led to increase in frataxin mRNA levels in patient cells (Fig. $2 a$ ) but not in control/unaffected cells (data not shown). Following anti-miR transfection we observed increase in frataxin protein levels (Fig. 2b) but not in another mitochondrial protein, cytochrome $c$ (data not shown). Thus, the anti-miR effect appears to be specific to FRDA-afflicted individuals and for frataxin as opposed to other nuclear encoded mitochondrial proteins such as cytochrome $c$. To decipher whether this effect of frataxin induction occurs at a transcriptional or post-transcriptional level, we used a transcription inhibitor actinomycin D in the presence of anti-miR-886-3p. The increase in FXN mRNA levels by the anti-miR was lost in the presence of transcription inhibitor, actinomycin D (Fig. 2c). Thus, anti-miR-886-3p acts transcriptionally to induce frataxin levels.

Histone deacetylase 3 has been shown to be involved in frataxin gene silencing in FRDA cells (Xu et al., 2009). Histone $\mathrm{H} 3 \mathrm{~K} 9$ dimethylation, a repressive mark is elevated at the first intron (Greene et al., 2007) and by contrast, H3K36 trimethylation, a mark associated with active chromatin has been shown to be reduced downstream of the GAA.TTC repeats in patient cells (Punga and Bühler, 2010). Surprisingly, in the presence of anti-miR886-3p, when frataxin levels were elevated, there was no change in histone deacetylase 3 levels (Fig. $2 d$ ), no reduction in repressive histone modification mark H3K9 dimethylation at the first intron (data not shown) and no increase in H3K36 trimethylation (a putative marker of gene activation) downstream of GAA.TTC repeats of the frataxin gene (data not shown). Thus, although the anti-miR-886-3p is exerting its effect at a transcriptional level, the two chromatin marks that we tested, classically associated with repression or activation, were unaltered upon frataxin induction. Of note, the histone deacetylase inhibitor $4 \mathrm{~b}$, which has been shown to increase frataxin levels in patient lymphoblasts (Herman et al., 2006) caused $\sim 50 \%$ decrease in the miR-886-3p levels (data not shown). This raises the interesting possibility that histone deacetylase (HDAC) inhibitors modulate miR-886-3p levels to derepress frataxin expression exclusive of or in addition to directly altering the

FRDA patients (Pianese et al., 2004). We did see elevated levels of miR-886-3p levels in eight patient peripheral blood samples that we tested (Fig. 1b). As we did in the case of miR-886, the remaining 26 miRNAs should be validated in additional patient samples to confirm their specific alteration in FRDA.

To check whether miR-886-3p directly regulates the frataxin levels we transfected control (GM08402) or patient cells (GM04078 and GM03816) with an anti-miR-886-3p. This trans- chromatin state at the frataxin gene locus.

\section{Discussion}

Here we show elevated levels of miR-886-3p in cells and blood from patients with FRDA compared with controls (Fig. 1b) where symptoms in patients are because of reduced frataxin levels (Pianese et al., 2004) due to GAA.TTC repeat expansion in first intron of frataxin gene. Transfection with anti-miR- 
886-3p led to increased frataxin levels in patient cells alone (Fig. 2a) and not in unaffected control cells (data not shown). This increase in frataxin levels was dependent on transcription as demonstrated by the ability of actinomycin D to suppress induction by anti-miR-886-3p (Fig. 2c). Interestingly a histone deacetylase inhibitor that has been shown to increase frataxin levels (Herman et al., 2006) caused decrease in the miR-886-3p levels. Histone deacetylase inhibitors have been shown previously to alter miRNA levels (Scott et al., 2006; Brest et al., 2011); however, this is the first link between HDAC inhibition and suppression of levels of miR-886-3p leading to enhanced frataxin expression (Fig. 3).

While intriguing, our findings do not exclude the possibility that the anti-miR effect on frataxin an indirect one via its effect on other proteins including erythropoietin (Epo). According to miRanda software, Epo is a target for miR-886 (John et al., 2004). Moreover, Epo has been shown to increase frataxin levels in FRDA patient cells and in clinical trials (Sturm et al., 2005; Boesch et al., 2008; Saccà et al., 2011). It is possible that the anti-miR transfection affects the Epo levels which in turn lead to increase in frataxin levels (Fig. 3). However, since the anti-miR effect was seen in skin fibroblasts which are not known to express Epo (Jelkmann, 2011), this is unlikely to be the exclusive mechanism for anti-miR effect we observe.

Recently pre-miR-886 has been designated as a vault RNA, VTRNA2, instead of a miRNA (Nandy et al., 2009; Stadler et al., 2009). These vault RNAs, transcribed by RNA polymerase III have been shown to produce regulatory small RNAs, svRNAs (small vault RNAs), by a Drosha independent, Dicerdependent mechanism (Persson et al., 2009). These regulatory small RNAs are $\sim 23 \mathrm{nt}$ in length, are capable of guiding sequence-specific cleavage of complementary target RNA and associate with Ago2. VTRNA2 has been shown to be processed into small regulatory RNA which in turn can regulate CYP3A4 gene expression in MCF7 cells via direct interaction with its 3' UTR (Persson et al., 2009). Since they were discovered in the 1980s, vault RNAs (Kedersha and Rome, 1986) have been implicated in resistance to certain drugs in cancer (Stadler et al., 2009), however, this is the first instance where we report its potentially causal association with a neurodegenerative disease. In April 2011, Lee et al. reported the analysis of pre-miR886 in cancer cells and concluded that this small RNA is neither a miRNA nor vault RNA (since it is not associated with vault particle) but acts as small noncoding regulatory RNA and is capable of physically interacting with $\sim 49$ proteins which include histone $\mathrm{H} 4$ and TNRC6A (Lee et al., 2011) suggesting other possible candidates for the effects of miR886-3p described here (Fig. 3).

Future studies will define precisely the mechanism by which miR-886-3p negatively regulates frataxin expression; in addition to the obvious therapeutic importance of anti-miR-886-3p, enhanced understanding of its mechanism of action may reveal new targets for intervention in FRDA.

\section{References}

Boesch S, Sturm B, Hering S, Scheiber-Mojdehkar B, Steinkellner H, Goldenberg H, Poewe W (2008) Neurological effects of recombinant human erythropoietin in Friedreich's ataxia: a clinical pilot trial. Mov Disord 23:1940-1944.

Brest P, Lassalle S, Hofman V, Bordone O, Gavric Tanga V, Bonnetaud C, Moreilhon C, Rios G, Santini J, Barbry P, Svanborg C, Mograbi B, Mari B, Hofman P (2011) MiR-129-5p is required for histone deacetylase inhibitor-induced cell death in thyroid cancer cells. Endocr Relat Cancer 18:711-719.

Campuzano V, Montermini L, Moltò MD, Pianese L, Cossée M, Cavalcanti F, Monros E, Rodius F, Duclos F, Monticelli A, Zara F, Cañizares J, Koutnikova H, Bidichandani SI, Gellera C, Brice A, Trouillas P, De Michele G, Filla A, De Frutos R, et al. (1996) Friedreich's ataxia: autosomal recessive disease caused by an intronic GAA triplet repeat expansion. Science 271:1423-1427.

Cogswell JP, Ward J, Taylor IA, Waters M, Shi Y, Cannon B, Kelnar K, Kemppainen J, Brown D, Chen C, Prinjha RK, Richardson JC, Saunders AM, Roses AD, Richards CA (2008) Identification of miRNA changes in Alzheimer's disease brain and CSF yields putative biomarkers and insights into disease pathways. J Alzheimers Dis 14:27-41.

Cossée M, Dürr A, Schmitt M, Dahl N, Trouillas P, Allinson P, Kostrzewa M, Nivelon-Chevallier A, Gustavson KH, Kohlschütter A, Müller U, Mandel JL, Brice A, Koenig M, Cavalcanti F, Tammaro A, De Michele G, Filla A, Cocozza S, Labuda M, et al. (1999) Friedreich's ataxia: point mutations and clinical presentation of compound heterozygotes. Ann Neurol 45:200-206.

Cossée M, Schmitt M, Campuzano V, Reutenauer L, Moutou C, Mandel JL, Koenig M (1997) Evolution of the Friedreich's ataxia trinucleotide repeat expansion: founder effect and premutations. Proc Natl Acad Sci U S A 94:7452-7457.

Greene E, Mahishi L, Entezam A, Kumari D, Usdin K (2007) Repeatinduced epigenetic changes in intron 1 of the frataxin gene and its consequences in Friedreich ataxia. Nucleic Acids Res 35:3383-3390.

Griffiths-Jones S (2004) The microRNA Registry. Nucleic Acids Res 32:D109-111.

Harding AE (1981) Friedreich's ataxia: a clinical and genetic study of 90 families with an analysis of early diagnostic criteria and intrafamilial clustering of clinical features. Brain 104:589-620.

Herman D, Jenssen K, Burnett R, Soragni E, Perlman SL, Gottesfeld JM (2006) Histone deacetylase inhibitors reverse gene silencing in Friedreich's ataxia. Nat Chem Biol 2:551-558.

Jelkmann W (2011) Regulation of erythropoietin production. J Physiol 589:1251-1258.

John B, Enright AJ, Aravin A, Tuschl T, Sander C, Marks DS (2004) Human MicroRNA targets. PLoS Biol 2:e363.

Johnson R, Zuccato C, Belyaev ND, Guest DJ, Cattaneo E, Buckley NJ (2008) A microRNA-based gene dysregulation pathway in Huntington's disease. Neurobiol Dis 29:438-445.

Kedersha NL, Rome LH (1986) Isolation and characterization of a novel ribonucleoprotein particle: large structures contain a single species of small RNA. J Cell Biol 103:699-709.

Kelly M, Bagnall RD, Peverill RE, Donelan L, Corben L, Delatycki MB, Semsarian C (2011) A polymorphic miR-155 binding site in AGTR1 is associated with cardiac hypertrophy in Friedreich ataxia. J Mol Cell Cardiol $51: 848-854$.

Lee K, Kunkeaw N, Jeon SH, Lee I, Johnson BH, Kang GY, Bang JY, Park HS, Leelayuwat C, Lee YS (2011) Precursor miR-886, a novel noncoding RNA repressed in cancer, associates with PKR and modulates its activity. RNA 17:1076-1089.

Montermini L, Andermann E, Labuda M, Richter A, Pandolfo M, Cavalcanti F, Pianese L, Iodice L, Farina G, Monticelli A, Turano M, Filla A, De Michele G, Cocozza S (1997) The Friedreich ataxia GAA triplet repeat: premutation and normal alleles. Hum Mol Genet 6:1261-1266.

Nandy C, Mrázek J, Stoiber H, Grässer FA, Hüttenhofer A, Polacek N (2009) Epstein-Barr virus-induced expression of a novel human vault RNA. J Mol Biol 388:776-784.

Persson H, Kvist A, Vallon-Christersson J, Medstrand P, Borg A, Rovira C (2009) The non-coding RNA of the multidrug resistance-linked vault particle encodes multiple regulatory small RNAs. Nat Cell Biol 11:1268-1271.

Pianese L, Turano M, Lo Casale MS, De Biase I, Giacchetti M, Monticelli A, Criscuolo C, Filla A, Cocozza S (2004) Real time PCR quantification of frataxin mRNA in the peripheral blood leucocytes of Friedreich ataxia patients and carriers. J Neurol Neurosurg Psychiatry 75:1061-1063.

Place RF, Li LC, Pookot D, Noonan EJ, Dahiya R (2008) MicroRNA-373 induces expression of genes with complementary promoter sequences. Proc Natl Acad Sci U S A 105:1608-1613. 
Punga T, Bühler M (2010) Long intronic GAA repeats causing Friedreich ataxia impede transcription elongation. EMBO Mol Med 2:120-129.

Saccà F, Piro R, De Michele G, Acquaviva F, Antenora A, Carlomagno G, Cocozza S, Denaro A, Guacci A, Marsili A, Perrotta G, Puorro G, Cittadini A, Filla A (2011) Epoetin alfa increases frataxin production in Friedreich's ataxia without affecting hematocrit. Mov Disord 26:739-742.

Scott GK, Mattie MD, Berger CE, Benz SC, Benz CC (2006) Rapid alteration of microRNA levels by histone deacetylase inhibition. Cancer Res 66:1277-1281.

Sonntag KC (2010) MicroRNAs and deregulated gene expression networks in neurodegeneration. Brain Res 1338:48-57.
Stadler PF, Chen JJ, Hackermüller J, Hoffmann S, Horn F, Khaitovich P, Kretzschmar AK, Mosig A, Prohaska SJ, Qi X, Schutt K, Ullmann K (2009) Evolution of vault RNAs. Mol Biol Evol 26:1975-1991.

Sturm B, Stupphann D, Kaun C, Boesch S, Schranzhofer M, Wojta J, Goldenberg H, Scheiber-Mojdehkar B (2005) Recombinant human erythropoietin: effects on frataxin expression in vitro. Eur J Clin Invest 35:711-717.

Tsou AY, Paulsen EK, Lagedrost SJ, Perlman SL, Mathews KD, Wilmot GR, Ravina B, Koeppen AH, Lynch DR (2011) Mortality in Friedreich ataxia. J Neurol Sci 307:46-49.

Xu C, Soragni E, Chou CJ, Herman D, Plasterer HL, Rusche JR, Gottesfeld JM (2009) Chemical probes identify a role for histone deacetylase 3 in Friedreich's ataxia gene silencing. Chem Biol 16:980-989. 